Published in final edited form as:

Nat Photonics. 2015 September 1; 9(9): 572-576. doi:10.1038/nphoton.2015.129.

\title{
Intracellular microlasers
}

\author{
Matjaž Humar ${ }^{1,2}$ and Seok Hyun Yun ${ }^{1,3,}$, \\ ${ }^{1}$ Wellman Center for Photomedicine, Harvard Medical School, Massachusetts General Hospital, \\ 65 Landsdowne St. UP-5, Cambridge, Massachusetts 02139, USA \\ ${ }^{2}$ Condensed Matter Department, J. Stefan Institute, Jamova 39, SI-1000 Ljubljana, Slovenia \\ ${ }^{3}$ Harvard-MIT Health Sciences and Technology, Cambridge, 77 Massachusetts Avenue \\ Cambridge, Massachusetts 02139, USA
}

\section{Abstract \\ Optical microresonators ${ }^{1}$ which confine light within a small cavity are widely exploited for various applications ranging from the realization of lasers ${ }^{2}$ and nonlinear devices ${ }^{3,4,5}$ to biochemical and optomechanical sensing $6,7,8,9,10,11$. Here we employ microresonators and suitable optical gain materials inside biological cells to demonstrate various optical functions in vitro including lasing. We explored two distinct types of microresonators: soft and hard, that support whispering-gallery modes (WGM). Soft droplets formed by injecting oil or using natural lipid droplets support intracellular laser action. The laser spectra from oil-droplet microlasers can chart cytoplasmic internal stress $\left(\sim 500 \mathrm{pN} / \mu^{2}\right)$ and its dynamic fluctuations at a sensitivity of 20 $\mathrm{pN} / \mu \mathrm{m}^{2}(20 \mathrm{~Pa})$. In a second form, WGMs within phagocytized polystyrene beads of different sizes enable individual tagging of thousands of cells easily and, in principle, a much larger number by multiplexing with different dyes.}

Luminescent probes including fluorescent dyes and proteins, quantum dots, bioluminescent molecules, and plasmonic nanoparticles have become indispensable tools in the fields of cell biology and medical sciences. While these molecular probes are immensely useful, their relatively broad emission spectra, typically in the range of 30 to $100 \mathrm{~nm}$, limit the number of probes simultaneously usable without ambiguity, and often make their spectra indistinguishable from broad background emission of endogenous molecules in tissues. It is fundamentally challenging to engineer molecules with much narrower spontaneous emission $^{12}$. However, photonic principles, such as optical resonance and stimulated emission, allow spectral narrowing (filtering) via successive coherent loss or gain. Generation of narrowband resonant emission from biological cells have been demonstrated by cellular lasers using external cavities ${ }^{13,14}$ or photonic crystal needles ${ }^{15}$. Recently, we

\footnotetext{
Reprints and permissions information is available online at www.nature.com/reprints.

*Corresponding author. syun@ hms.harvard.edu.

Author Contributions

M.H. and S.H.Y. designed the study. M.H. carried out the experiments and analysed the data. M.H. and S.H.Y. wrote the manuscript.

Supplementary information is available in the online version of the paper.

Competing financial interests

The authors declare no competing financial interests.
} 
have sought to generate stand-alone cell lasers ${ }^{16}$, and herein describe effective approaches based on intracellular whispering-gallery mode (WGM) micro-resonators formed by soft and hard polymeric materials. WGMs are formed when light is circulating in a transparent spherical object by being trapped due to total internal reflection at the interface. WGM cavities can have sizes at the micro- and nano-scale ${ }^{17}$ although they are much larger than the conventional luminescent probes.

We first describe soft WGM cavities in the form of oil droplets in cells. We injected nile red dye-mixed polyphenyl ether (PPE), a chemically inert optical grade fluid with low viscosity $(100 \mathrm{cP})$ and a refractive index (n) of 1.69 (Fig. 1a). The size of the droplets was controlled $^{18}$ in a range of 4 to $20 \mu \mathrm{m}$, corresponding to a volume of $30 \mathrm{fL}$ to $4000 \mathrm{fL}$ (Supplementary Video 1 and Fig. 1b). Droplets larger than $7 \mu \mathrm{m}$ show lasing upon pulsed excitation ( $\lambda=535 \mathrm{~nm}, 5 \mathrm{~ns}, 10 \mathrm{~Hz}$ ) with thresholds as low as a few nanojoules per pulse (Fig. 1c-d). This energy level is non-harmful for the cell ${ }^{13}, 14$. The instantaneous heating of a droplet is calculated to be $<1^{\circ} \mathrm{C}$, and the ambient temperature increase in the cytosol is negligible (see Supplementary Information). When the droplet is under uniaxial stress, its shape deviates from a sphere, and the deformation is manifested in the emission spectrum as splitting of laser lines (Fig. 1e). For small deformation, the shape can be approximated as a spheroid, which supports laser oscillation in the equatorial plane, which has the lowest curvature and, therefore, minimum optical loss. The modes are fitted to a model, and the equatorial and polar semi-axes, $a$ and $b$, respectively, are determined (see Supplementary Information). From Laplace's law, the flattening stress, $\Delta \sigma$, is related to the local mean curvature of droplet surface ${ }^{19}: \Delta \sigma=2 \gamma \Delta H$ is surface tension and $\Delta H$ is the difference in the curvature (see Supplementary Information). For small eccentricity $(e)$, i.e. $e^{2}<<1$, the stress is approximated as:

$$
\Delta \sigma \approx \frac{2 \gamma}{a} e^{2}
$$

For the droplet in Fig. 1e, we measure $a=8.5 \mu \mathrm{m}$ and $b=8.3 \mu \mathrm{m}$; and with $\gamma \approx 45 \mathrm{mN} / \mathrm{m}$ for $\mathrm{oil} /$ water interface, we determine $\Delta \sigma=500 \mathrm{pN} / \mu \mathrm{m}^{2}$. Time-lapse traces of the output spectra revealed dynamic variations of the cellular stress in live cells (Fig. 1f). The mean fluctuation of the internal stress was measured to be $\sim 0.15 \mathrm{nN} / \mu^{2}{ }^{2}$ (Fig. $1 \mathrm{~g}$ ). From the baseline fluctuation in dead cells $\left(\left\langle\Delta e^{2}\right\rangle=0.19 \%\right)$, the force sensitivity is $\sim 20 \mathrm{pN} / \mu \mathrm{m}^{2}(20 \mathrm{~Pa})$, which is an order of magnitude better than direct image-based analysis ${ }^{19}$.

We next explored if cells naturally containing lipid droplets ( $\mathrm{n}=1.47)$ can support laser oscillation (Fig. 2a). Adipocytes freshly extracted from porcine subcutaneous tissue contain a single lipid droplet with nearly perfect spherical geometry (Fig. 2b-c). After incubation with a lipophilic fluorescent dye and pumping with a pulsed laser, the cells exhibited lasing with WGM outputs (Fig. 2d) and distinct threshold (Fig. 2e). This represents a completely natural intracellular optical cavity. Next, we investigated the possibility of generating lasing from adipocytes in situ in tissues. Adipocytes in fat are closely packed and have random shapes (Fig. 2f), which have lower cavity Q-factor and require higher pump energy for lasing. To lower threshold, we injected a mixture of collagenase and lipophilic nile red dye into the subcutaneous fat. The collagenase releases adipocytes from the tissue matrix, so that 
they acquire spherical shapes ${ }^{20}$. An optical fibre was inserted through a needle puncture hole to excite the adipocytes with pulsed laser light and to collect the light emitted from the tissue (Fig. 2g). The adipocytes near the fibre tip readily showed lasing (Fig. 2h). In some cases also adipocytes at the rim of fat tissue that had more round shape showed lasing (Supplementary Fig. 1) eliminating the need of collagenase.

Solid microspheres, such as polystyrene microspheres, offer a simple way to devise nondeformable, intracellular lasers (Fig. 3a). Polystyrene beads are readily internalized by endocytosis ${ }^{21,22}$ (Fig. 3b). We have observed that both macrophages and non-phagocytic cells, such as HeLa and NIH3T3, engulf beads up to $20 \mu \mathrm{m}$ in diameter $(d)$, large enough to exhibit lasing at low pump energy. Viability of HeLa cells $24 \mathrm{~h}$ after they engulfed one or more polystyrene beads ( $>6 \mu \mathrm{m}$ ) was $98.4 \% \pm 0.6 \%$, compared to $99.4 \% \pm 0.2 \%$ for cells without beads. WGM lasers offer multiple options for the position of the gain medium, including inside the resonator, outside the resonator, and on the surface of the bead. We tested these three cases. First, fluorescent dye-embedded polystyrene beads provided gain within the beads. Beads larger than $d=11 \mu \mathrm{m}$ supported lasing inside cells at pump energy levels below a few nanojoules (Fig. 3c and d, Supplementary Fig. 2). The bead surface can be functionalized with probe molecules, such as antibodies or deoxyribonucleic acid (DNA), for intracellular molecular sensing 6, 7, 8, 9, 10,11. Second, a cell tracker dye, 5chloromethylfluorescein diacetate (CMFDA), retained in the cytosol served as the gain medium for $\mathrm{BaTiO}_{3}$ beads. Lasing down to $8 \mu \mathrm{m} \mathrm{BaTiO}_{3}$ beads was observed (Fig. 3e). The gain medium interacts freely with the cell, generating amplified signals modulated by the cavity resonance. Thirdly, the surface of high-index $(n=1.9) \mathrm{BaTiO}_{3}$ beads was coated with fluorescent dye (Alexa Fluor 488). Although a monolayer of dye did not provide enough gain for lasing, its emission spectrum is strongly modulated by high-Q (700) resonance in beads down to $d=3.5 \mu \mathrm{m}$, with relatively low fluorescence background (Fig. 3f). Any combinations of the three gain locations are of course possible. Furthermore, the gain and sensing media may be collocated or separated.

The precise wavelengths of the multiple spectral peaks from a bead above or below lasing threshold (Fig. 3) are uniquely determined from the size of the bead. We measured the output spectra from fluorescent polystyrene beads from each spatial location in a mid-plane of the cell (Fig. 4a) in a confocal hyperspectral imaging setup with numerical aperture of 1.25 and continuous-wave pumping with a 455-nm light emitting diode (LED). The spatial map of the intensity of resonance peaks shows a ring shape (Fig. 4b), representing light circulating in the bead and leaking out in the tangential direction. By fitting the spectra with WGM mode theory ${ }^{23}$, the effective bead diameter $(d)$ was calculated at each pixel. The diameter around circumference varies by $1-2 \mathrm{~nm}$, which may reflect the deviation of the actual bead from a perfect sphere (Fig. 4c). The precision of the mean diameter is about 50 pm (Supplementary Fig. 3), which represents a remarkable sensitivity of WGM analysis (50 $\mathrm{pm} / 7.7 \mu \mathrm{m}=6.5 \times 10^{-6}$ ). The same type of analysis can be done for beads operated above threshold by pulsed pumping. However, when the number of lasing modes is less than 3-4, the size measurement is frustrated or has an error as much as 1-2 free spectral ranges ( 100 nm) (Supplementary Fig. 4). 
An intriguing application of this precision measurement is to use beads with different diameters to tag individual cells (Fig. 4d-e, Supplementary Fig. 5, and Supplementary Video 2). The diameter interval between beads should be large enough to accommodate the typical wavelength variation of $2 \mathrm{~nm}$ due to refractive index changes by intracellular dynamics $(\Delta \lambda / \lambda \approx \Delta n / n+\Delta d / d)$, where $n$ is effective refractive index for the oscillating mode). With polydispersed beads in a range from 8 to $12 \mu \mathrm{m}$ and a bin size of $2 \mathrm{~nm}$, we can distinguish 2,000 individual beads. By using different fluorescent dyes with distinct emission bands this number can be increased easily by several folds. Furthermore, each cell can engulf multiple particles (Supplementary Fig. 6b). Using three beads per cell and 5 different fluorescence dyes with non-overlapping spectra, it would be possible to individually tag ${ }^{2000 \times 5} C_{3}=$ $2 \times 10^{11}$ cells, which is comparable to the number of entire cells in the human body and many orders of magnitude more than state-of-the-art techniques such as stochastic brainbow recombination ${ }^{24}$ for in vivo cell tracking, as well as high-throughput on-chip cytometry and cell-based analysis.

To demonstrate intracellular sensing, we measured the spectral changes of Alexa Fluor 488 coated soda lime glass beads in HeLa cells while the cell culture media was supplied with an additional $2 \mathrm{~g} / \mathrm{L}$ of sodium chloride. Exposure of cells to hypertonic solution causes intracellular water to quickly diffuse out of the cell, reducing the cell volume. The shrinking is followed by a partial recovery by regulatory volume increase ${ }^{25}$. The change in cell volume changes the concentration of the molecules in the cytoplasm therefore also changing the refractive index. The time dependence of the shift in the position of a WGM (Fig. 4f) corresponds to $\Delta \mathrm{n} \approx 1.0 \times 10^{-2}$, which is close to the calculated value of $7.6 \times 10^{-3}$ from Boyle-van't Hoff law (see Supplementary Information). As a control experiment, adding the same volume of media without changing the osmolarity produces no response (Supplementary Fig. 7).

Here we have demonstrated stand-alone cell lasers by employing an intracellular WGM microresonator, dye gain medium, and far-field optical pumping with nanosecond pulses. Pulse duration of $100 \mathrm{ps}$ could reduce threshold pump energy (by $\sim 50$ times) while supporting distinct WGM modes in microresonators. The shape of intracellular WGM cavities is not limited to spheres. Cylinders, torus and disks can also be used. The micrometre size may offer flexibility in cavity engineering for specific mode selection or direction-dependent radiation ${ }^{26}$. Biodegradable polymers ${ }^{27}$ may improve biocompatibility. Smaller cavities causing minimal perturbations on cells would also be useful. This requires high refractive index materials (Fig. 5). Semiconductor materials ( $\mathrm{n}>3$ ) should enable submicron WGM lasers ${ }^{28}$. Realization of metamaterials with extremely high refractive index ${ }^{29}$ could also enable sub-micron lasers. Plasmon-based spasers can be as small as tens of nanometres $^{30}$, but WGM analysis demonstrated here are most effective with microresonators accommodating multiple optical wavelengths.

\section{Methods}

\section{Optical setup}

For the pumping of the cell lasers and the collection of light, a $40 \times 1.25 \mathrm{NA}$ or a $100 \times 1.40$ NA oil immersion objective was used. The pumping was achieved using an optical 
parametric oscillator with $5 \mathrm{~ns}$ pulse duration, tuned to $475 \mathrm{~nm}$ for green dyes or $535 \mathrm{~nm}$ for red dyes. The laser beam was slightly divergent at the objective entrance pupil, so that the focus at the sample was located slightly further away from the objective focal plane; the beam diameter at the objective focal plane was approximately $20 \mu \mathrm{m}$ wide. For measurements of polystyrene microsphere modes below laser threshold (Fig. 4), a $455 \mathrm{~nm}$ LED was used as an excitation source. The collected light was sent through a dichroic mirror and split 50:50 to CCD camera and imaging spectrometer (300 mm focal length, 0.05 $\mathrm{nm}$ resolution). For all the measurements except for hyperspectral imaging, the spectrum was collected through the entrance slit of the spectrometer and therefore represents an integration along a line crossing the centre of a bead or droplet. For hyperspectral imaging the spectrometer slit was replaced by a $10-\mu \mathrm{m}$ pinhole, and a $2 \mathrm{D}$ raster scan was performed with $0.1 \mathrm{~s}$ per pixel acquisition time. The spectral peaks above a broad fluorescence background were integrated to get the laser intensity image shown in Fig. 4b. The individual spectral peaks after subtracting the background were fitted with a Lorentzian curve to get their central wavelengths, which were subsequently fitted to equation (1), with $a=b$.

\section{Cell culture}

HeLa, NIH3T3 and RAW 264.7 cell lines were grown at $37^{\circ} \mathrm{C}$ with $5 \% \mathrm{CO} 2$ in full growth medium (Dulbecco's Modified Eagle Medium supplemented with 10\% fetal bovine serum and $1 \%$ pen-strep). The cells were incubated in full growth medium supplied with beads for $12 \mathrm{~h}$ before laser experiments. The viability assay was performed $24 \mathrm{~h}$ after the cells were injected with PPE or they were supplied with beads using ethidium homodimer-1.

\section{Oil injection}

Non-toxic, low-viscosity high-index polyphenyl ether oil (PPE, SL-5267, Santolubes) was doped with $5 \mathrm{mM}$ nile red (9-diethylamino-5-benzo[a]phenoxazinone) prior to its injection into HeLa and NIH3T3 cells. The injection was performed using a microinjector (FemtoJet, Eppendorf) and a glass micropipette with $1.0 \mu \mathrm{m}$ outer diameter (Femtotip, Eppendorf). The size of the injected droplets was controlled by the injection time ranging from $0.2 \mathrm{~s}$ to $1 \mathrm{~s}$, with an injecting pressure of $1700 \mathrm{kPa}$.

\section{Adipocytes}

Fresh subcutaneous fat tissue was collected from the neck of a two-month old pig, minced and mixed with equal volume of phosphate buffered saline (PBS) supplied with $2 \mathrm{mg} / \mathrm{mL}$ collagenase (type 1A). The mixture was incubated with frequent shaking at $37^{\circ} \mathrm{C}$ for 30 min. The suspension was filtered through $250 \mu$ m nylon mesh to remove undigested tissue and centrifuged at $65 \mathrm{~g}$ for $5 \mathrm{~min}$ to collect top layer of fat. The adipocytes were stained by adding $1 \%$ of $10 \mathrm{mM}$ nile red in acetone. In the tissue laser experiments, $1 \mathrm{~mL}$ of PBS containing $1 \mathrm{mg} / \mathrm{mL}$ collagenase and $1 \%$ of $10 \mathrm{mM}$ nile red in acetone was injected in the subcutaneous porcine fat. The tissue was incubated at $37^{\circ} \mathrm{C}$ for $15 \mathrm{~min}$. For excitation and light collection a multimode fiber with core diameter $200 \mu \mathrm{m}$ was used. 


\section{Fluorescent beads}

Three different bead types were used: $8 \mu \mathrm{m}$ mean diameter green fluorescent polystyrene spheres (Thermo Scientific, Fluoro-Max, 18\% coefficient of variation), 15-19 $\mu \mathrm{m}$ soda lime glass beads (Cospheric), and polydisperse $\mathrm{BaTiO}_{3}$ beads with a broad size distribution of 1-40 $\mu \mathrm{m}$ (GL0175B, Mo-Sci). Polystyrene beads were incubated for $30 \mathrm{~min}$ at room temperature in $1 \mathrm{wt} \%$ poly-L-lysine hydrobromide (MW 30,000-70,000) in water and washed three times. Soda lime and $\mathrm{BaTiO}_{3}$ spheres were coated with Alexa Fluor 488 tetrafluorophenyl (TFP) ester dye (Life Technologies) as follows. Beads were washed with acetone and incubated in acetone containing $2 \% \mathrm{v} / \mathrm{v} 3$-aminopropyltriethoxysilane for 15 min with mixing. The beads were washed two times with acetone, one time with water and dried at $120{ }^{\circ} \mathrm{C}$ for $30 \mathrm{~min}$. The beads were re-dispersed in $0.1 \mathrm{M}$ sodium bicarbonate buffer, $\mathrm{pH}$ 9.0. $100 \mu \mathrm{g}$ of Alexa Fluor TFP ester dissolved in $10 \mu \mathrm{L}$ dimethyl sulfoxide was added to $200 \mu \mathrm{L}$ of bead dispersion in the sodium bicarbonate buffer. After $2 \mathrm{~h}$ incubation, the beads were washed five times with water and transferred to phosphate buffered saline. All the above washing steps were performed by centrifugation at $5000 \mathrm{~g}$ for $5 \mathrm{~min}$ and exchange of the medium.

\section{Supplementary Material}

Refer to Web version on PubMed Central for supplementary material.

\section{Acknowledgements}

This research was supported in part by the U.S. National Science Foundation (ECCS-1101947, EEC-1358296, ECCS-1505569) and National Institutes of Health (P41 EB015903). M.H. was supported in part by the Marie Curie International Outgoing Fellowship $\mathrm{N}^{\circ} 627274$ within the $7^{\text {th }}$ European Community Framework Programme. The authors thank Jie Zhao and Wellman Centre Photopathology Core for technical support. Part of this work was performed at the Centre for Nanoscale Systems (CNS) in Harvard University, which is a member of the National Nanotechnology Infrastructure Network (NNIN) and supported by the National Science Foundation under NSF award no. ECS-0335765.

\section{References and Notes}

1. Vahala KJ. Optical microcavities. Nature. 2003; 424:839-846. [PubMed: 12917698]

2. Qian SX, Snow JB, Tzeng HM, Chang RK. Lasing droplets - highlighting the liquid-air interface by laser-emission. Science. 1986; 231:486-488. [PubMed: 17776021]

3. Hill MT, et al. A fast low-power optical memory based on coupled micro-ring lasers. Nature. 2004; 432:206-209. [PubMed: 15538365]

4. Kippenberg TJ, Holzwarth R, Diddams SA. Microresonator-based optical frequency combs. Science. 2011; 332:555-559. [PubMed: 21527707]

5. Spillane SM, Kippenberg TJ, Vahala KJ. Ultralow-threshold Raman laser using a spherical dielectric microcavity. Nature. 2002; 415:621-623. [PubMed: 11832940]

6. Armani AM, Kulkarni RP, Fraser SE, Flagan RC, Vahala KJ. Label-free, single-molecule detection with optical microcavities. Science. 2007; 317:783-787. [PubMed: 17615303]

7. Vollmer F, Arnold S. Whispering-gallery-mode biosensing: label-free detection down to single molecules. Nat. Methods. 2008; 5:591-596. [PubMed: 18587317]

8. Zhu JG, et al. On-chip single nanoparticle detection and sizing by mode splitting in an ultrahigh-Q microresonator. Nat. Photonics. 2010; 4:46-49.

9. Fan XD, White IM. Optofluidic microsystems for chemical and biological analysis. Nat. Photonics. 2011; 5:591-597. [PubMed: 22059090] 
10. Baaske MD, Foreman MR, Vollmer F. Single-molecule nucleic acid interactions monitored on a label-free microcavity biosensor platform. Nat. Nanotechnol. 2014; 9:933-939. [PubMed: 25173831]

11. Himmelhaus M, Francois A. In-vitro sensing of biomechanical forces in live cells by a whispering gallery mode biosensor. Biosens. Bioelectron. 2009; 25:418-427. [PubMed: 19699629]

12. Zhang J, Campbell RE, Ting AY, Tsien RY. Creating new fluorescent probes for cell biology. Nat. Rev. Mol. Cell Biol. 2002; 3:906-918. [PubMed: 12461557]

13. Gather MC, Yun SH. Single-cell biological lasers. Nat. Photonics. 2011; 5:406-410.

14. Jonáš A, et al. In vitro and in vivo biolasing of fluorescent proteins suspended in liquid microdroplet cavities. Lab. Chip. 2014; 14:3093-3100. [PubMed: 24968888]

15. Shambat G, et al. Single-cell photonic nanocavity probes. Nano Lett. 2013; 13:4999-5005. [PubMed: 23387382]

16. Fan X, Yun S-H. The potential of optofluidic biolasers. Nat. Methods. 2014; 11:141-147. [PubMed: 24481219]

17. Hill MT, Gather MC. Advances in small lasers. Nat. Photonics. 2014; 8:908-918.

18. Zhang Y, Yu LC. Microinjection as a tool of mechanical delivery. Curr. Opin. Biotechnol. 2008; 19:506-510. [PubMed: 18725294]

19. Campàs O, et al. Quantifying cell-generated mechanical forces within living embryonic tissues. Nat. Methods. 2014; 11:183-189. [PubMed: 24317254]

20. Gardan D, Gondret F, Louveau I. Lipid metabolism and secretory function of porcine intramuscular adipocytes compared with subcutaneous and perirenal adipocytes. Am. J. Physiol. Endocrinol. Metabol. 2006; 291:E372-E380.

21. Cannon GJ, Swanson JA. The macrophage capacity for phagocytosis. J. Cell Sci. 1992; 101:907913. [PubMed: 1527185]

22. Kobayashi S, et al. Artificial induction of autophagy around polystyrene beads in nonphagocytic cells. Autophagy. 2010; 6:36-45. [PubMed: 19901555]

23. Gorodetsky ML, Fomin AE. Geometrical theory of whispering-gallery modes. IEEE J. Sel. Topics Quantum Electron. 2006; 12:33-39.

24. Livet J, et al. Transgenic strategies for combinatorial expression of fluorescent proteins in the nervous system. Nature. 2007; 450:56-62. [PubMed: 17972876]

25. Schultz, SG. Molecular biology of membrane transport disorders. Springer; 1996.

26. Wang QJ, et al. Whispering-gallery mode resonators for highly unidirectional laser action. Proc. Natl. Acad. Sci. U. S. A. 2010; 107:22407-22412. [PubMed: 21149678]

27. Nizamoglu S, Gather MC, Yun SH. All-biomaterial laser using vitamin and biopolymers. Adv. Mater. 2013; 25:5943-5947. [PubMed: 24425626]

28. Zhang Z, et al. Visible submicron microdisk lasers. Appl. Phys. Lett. 2007; 90:111119111119-111113.

29. Choi M, et al. A terahertz metamaterial with unnaturally high refractive index. Nature. 2011; 470:369-373. [PubMed: 21331038]

30. Stockman MI. Spasers explained. Nat. Photonics. 2008; 2:327-329. 

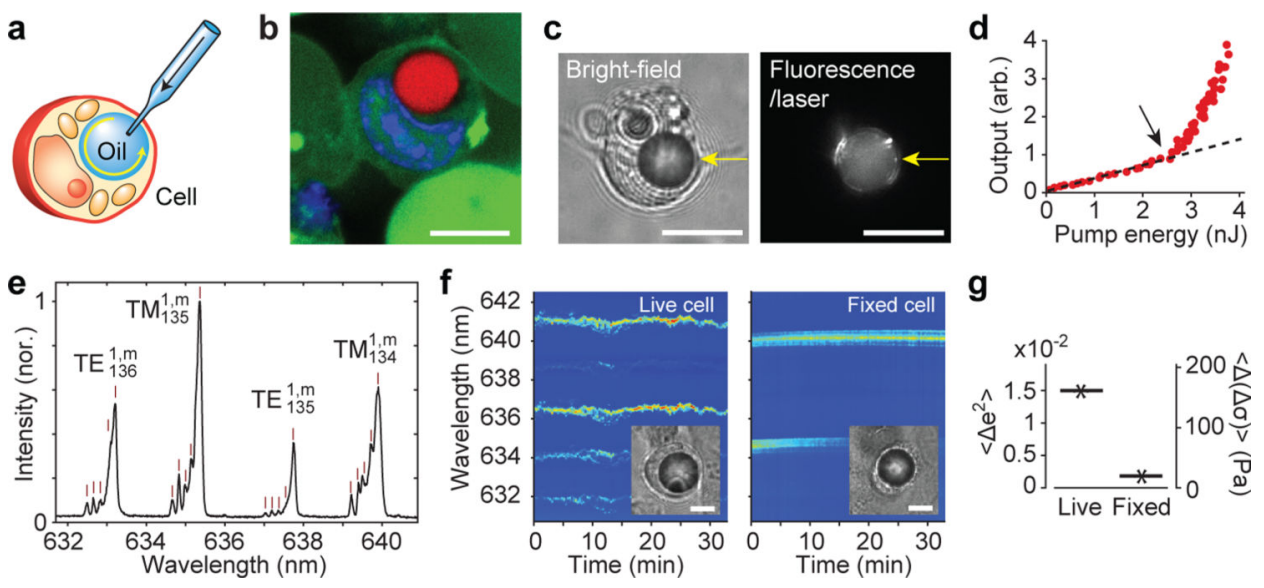

g

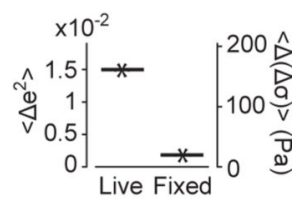

Figure 1. Injected oil droplet laser

a, Illustration of the injection of oil into the cytoplasm of a cell. $\mathbf{b}$, Confocal fluorescence image of a cell with a PPE droplet doped with nile red dye (red). The cell nucleus (blue) became a kidney-shaped form, giving space to the droplet. c, Bright-field (left) and laseroutput (right) images of a cell with a droplet (arrow) above the lasing threshold. d, Output light intensity from a droplet as a function of pump pulse energy, showing a distinct laser threshold (arrow). Dotted line, linear fit to the fluorescence output below the threshold. e, A typical output spectrum of the lasing modes. All the modes are first radial modes, two modes with TE and two with TM polarization. Each mode is split into multiple submodes. From their splitting in this data, the spheroid is determined to be of oblate shape with equatorial and polar semi-axes 8.3 and $8.5 \mu \mathrm{m}$ respectively. $\mathbf{f}$, Time-lapse variation of the output spectrum for a live cell (left) and a dead cell fixed with formaldehyde (right). g, Standard deviation of the square of eccentricity and corresponding internal stress for live and fixed (dead) cells. Scale bars, $10 \mu \mathrm{m}$ in b-c and f. 

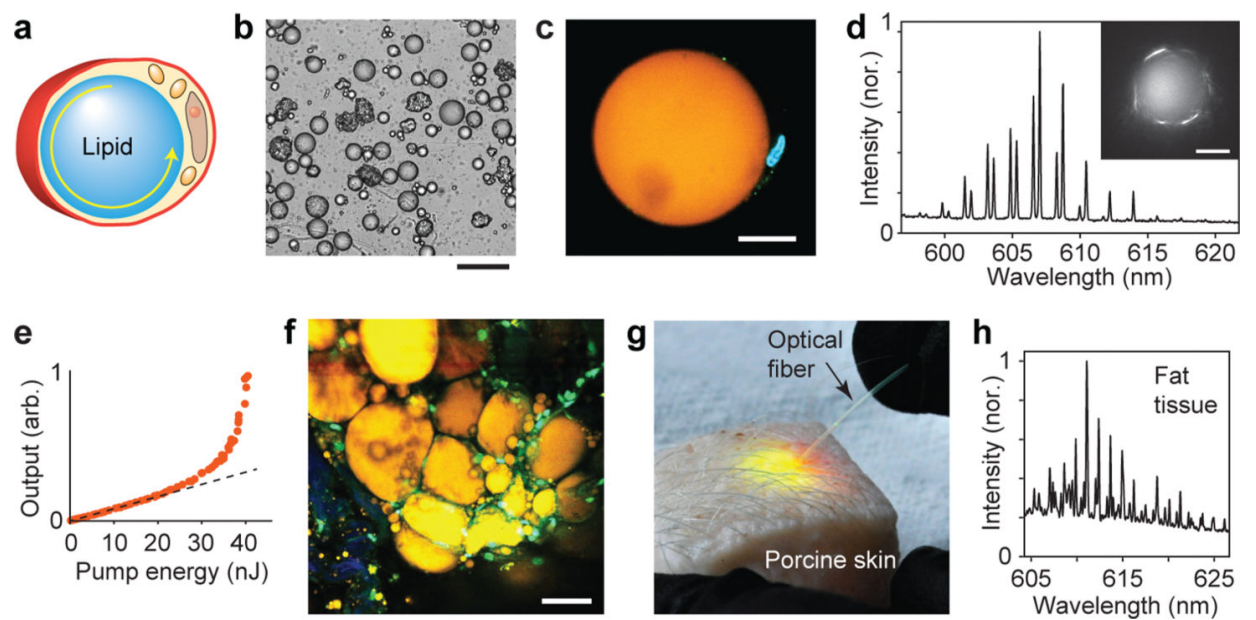

Figure 2. Adipocyte lasers

a, Illustration of a typical mature subcutaneous adipocyte with a lipid droplet. b, Individual adipocytes extracted from subcutaneous porcine fat. c, A confocal image of an adipocyte containing a large lipid droplet (orange), occupying the majority of the cell volume. The nucleus (blue) is visible next to the droplet. Scale bar, $20 \mu \mathrm{m}$. d, Spectrum from a $45 \mu \mathrm{m}$ adipocyte above lasing threshold, showing typical WGM spectral peaks. Inset, a fluorescence image of the cell above lasing threshold. e, Output energy as a function of the pump energy. Dotted line, linear fit to the fluorescence output below the laser threshold. $\mathbf{f}$, Two-photon confocal image of adipocytes in situ in a subcutaneous fat tissue, after intradermal injection of nile red dye (yellow). $\mathbf{g}$, Generating cellular laser emission from within tissue. The pump is fibre-optically into the subcutaneous fat layer after injecting a mixture of collagenase and nile red dye. $\mathbf{h}$, The spectrum of light collected by the optical fibre from the tissue. Scale bars, $200 \mu \mathrm{m}$ in $\mathbf{b}, 20 \mu \mathrm{m}$ in $\mathbf{c}, \mathbf{d}$ and $\mathbf{f}$. 
a

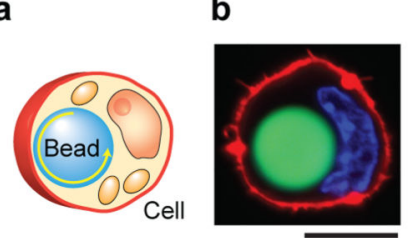

b

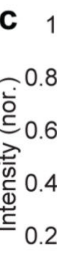
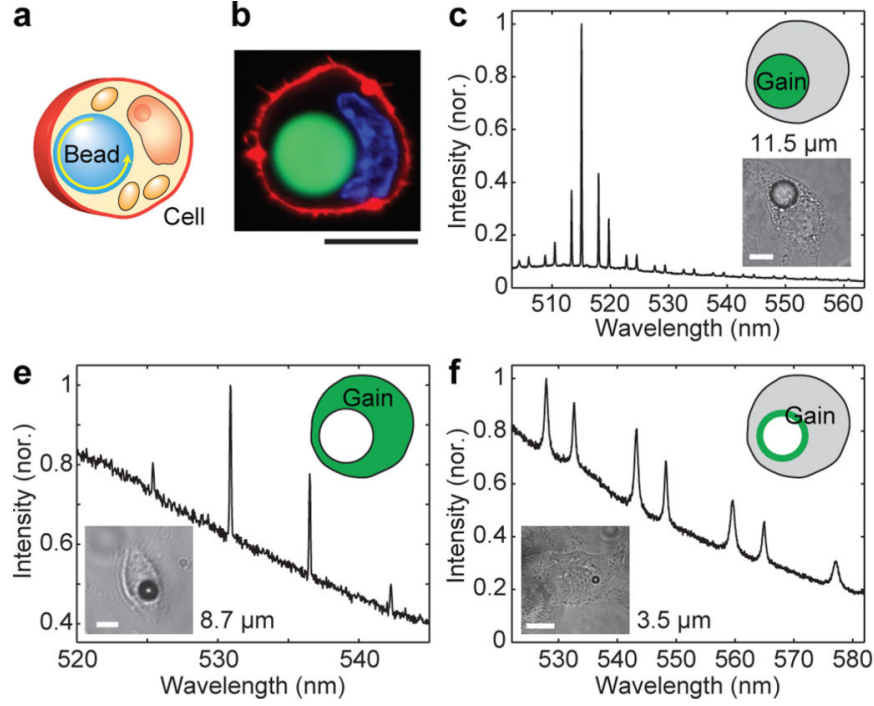

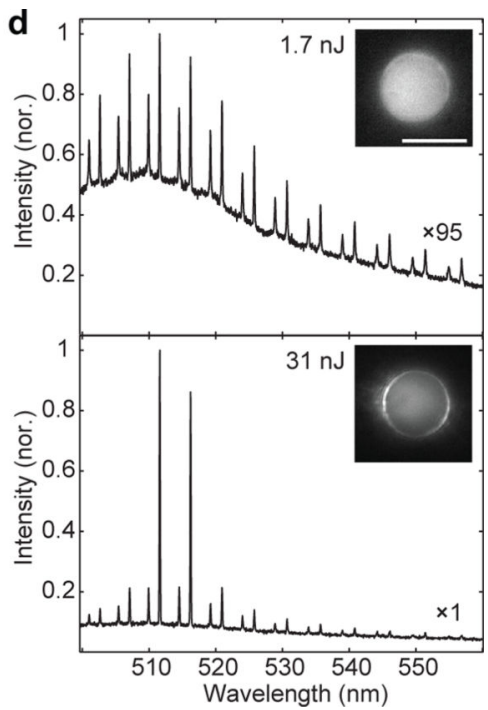

Figure 3. Three different types of solid intracellular microcavities

a, Illustration of a bead inside a cell. b, Confocal fluorescence image of a HeLa cell containing a polystyrene bead (green), nucleus (blue) and plasma membrane (red). c, Laser emission from a fluorescent polystyrene bead inside a cell. d, Emission spectra and images (insets) of a fluorescent polystyrene bead below and above lasing threshold (3.2 nJ). e, Laser output from a $8.7 \mu \mathrm{m}$ non-fluorescent $\mathrm{BaTiO}_{3}$ bead embedded in a cell that contains CMFDA dye in its cytoplasm. f, Spontaneous emission from a $3.5 \mu \mathrm{m} \mathrm{BaTiO}_{3}$ bead coated with Alexa 488 dye below laser threshold. Scale bars, $10 \mu \mathrm{m}$. 

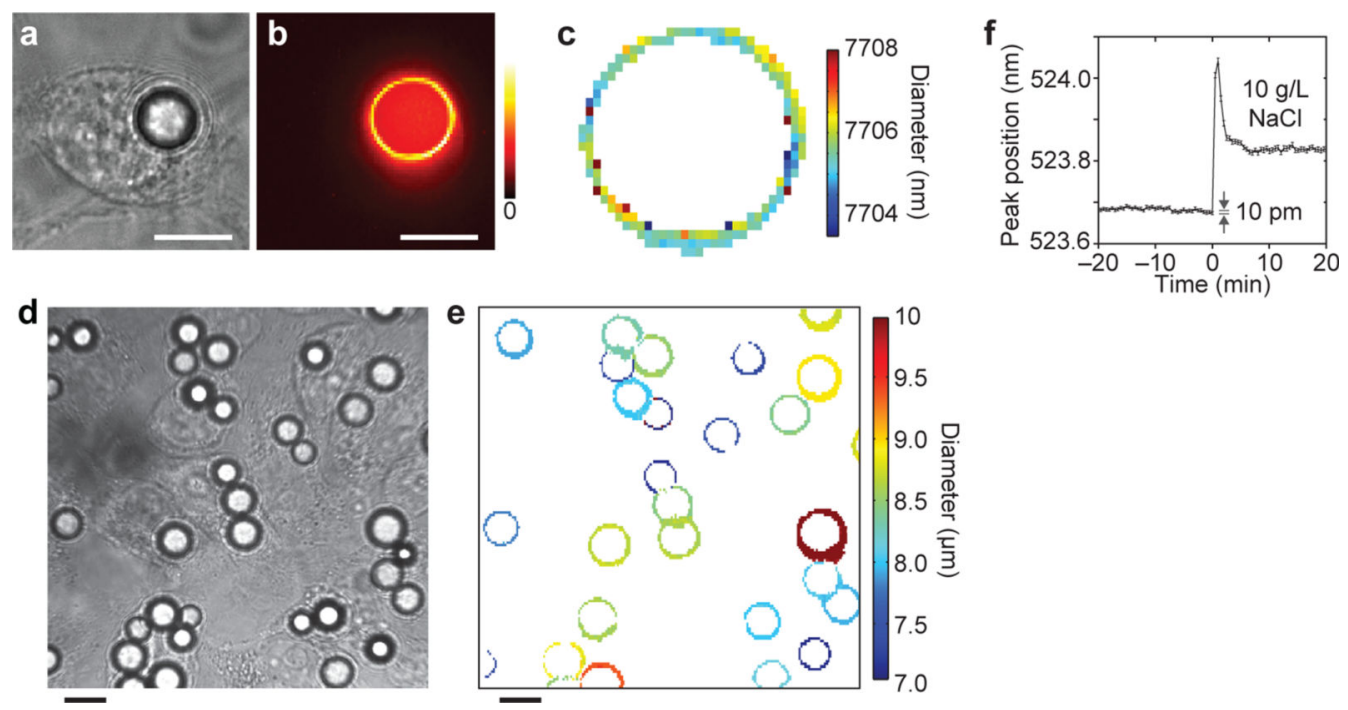

Figure 4. Tagging and sensing applications of solid intracellular microcavities

a, Bright field image of a HeLa cell containing a polystyrene fluorescent bead. b, False colour image of the cell, representing intensity of the oscillating WGMs. c, A bead diameter map calculated from confocal hyperspectral images of the WGM output. d, Multiple HeLa cells containing beads and $\mathbf{e}$, the corresponding bead diameter map. $\mathbf{f}$, Time-lapse measurement of a resonant peak from a glass bead inside a HeLa cell. The addition of $2 \mathrm{~g} / \mathrm{L}$ of sodium chloride at $\mathrm{t}=0$ caused the peak wavelength to shift. Scale bars, $10 \mu \mathrm{m}$. 


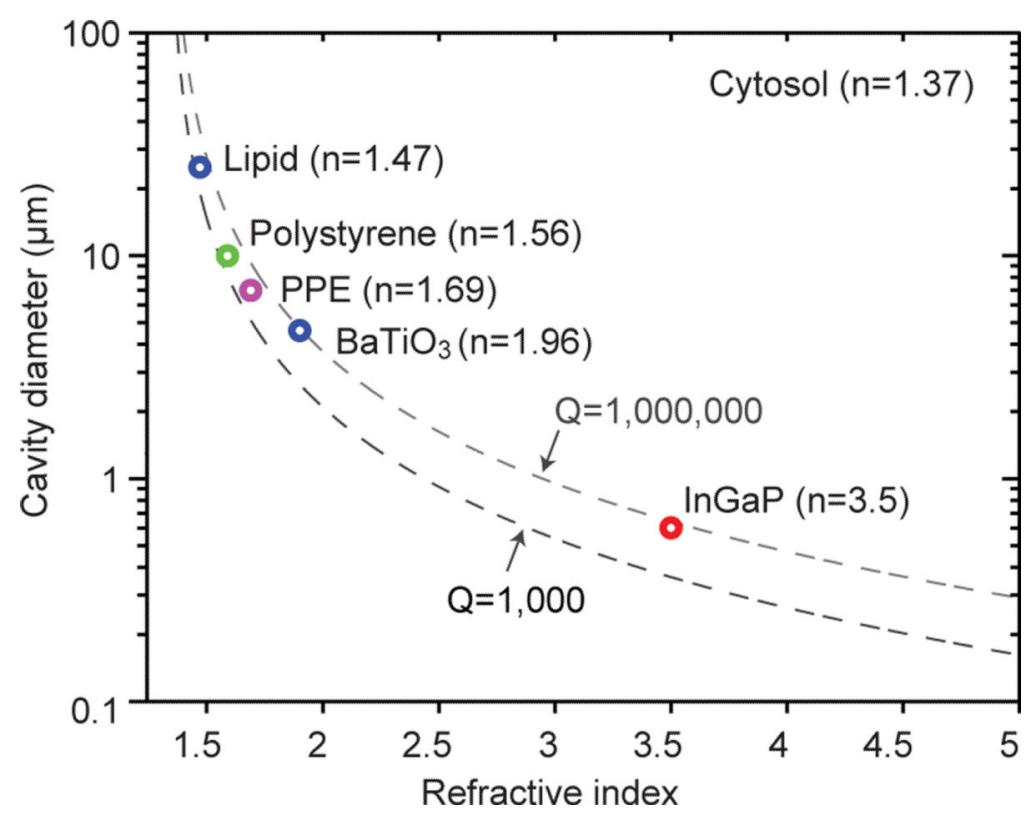

Figure 5. Size of intracellular microlasers

Q-factors of WGMs are highly dependent on the refractive index and cavity size. The two dashed lines represent theoretical calculation of two Q-factors of $10^{6}$ and $10^{3}$, respectively. The circles represent the measured minimum size of laser achieved inside a cell for lipid droplet in an adipocyte, polystyrene bead and PPE droplet inside a HeLa cell, and $\mathrm{BaTiO}_{3}$ beads in $15 \mathrm{mM}$ Pyrromethene solution, as well as InGaP disc lasers in the air ${ }^{28}$. 